\title{
Abnormal findings on digital vectoelectronystagmography in patients with temporomandibular disorders
}

\author{
Adriana Tresso ${ }^{1}$ \\ https://orcid.org/0000-0001-8311-7359 \\ Gustavo José Luvizutto 1 \\ https://orcid.org/0000-0002-6914-7225 \\ Rodrigo Bazan ${ }^{2}$ \\ https://orcid.org/0000-0003-3872-308X \\ Heloisa Helena Caovilla ${ }^{3}$ \\ https://orcid.org/0000-0003-4157-8995 \\ Maurício Malavasi Ganança ${ }^{3}$ \\ https://orcid.org/0000-0003-4483-8502
}

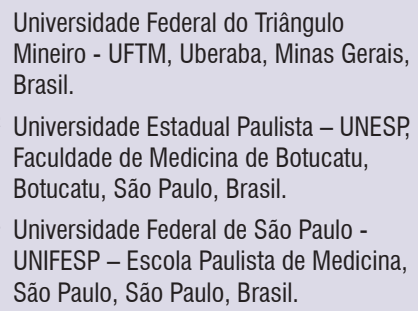

Universidade Estadual Paulista - UNESP, Faculdade de Medicina de Botucatu, Botucatu, São Paulo, Brasil.

Universidade Federal de São Paulo UNIFESP - Escola Paulista de Medicina, São Paulo, São Paulo, Brasil.

Conflict of interests: Nonexistent

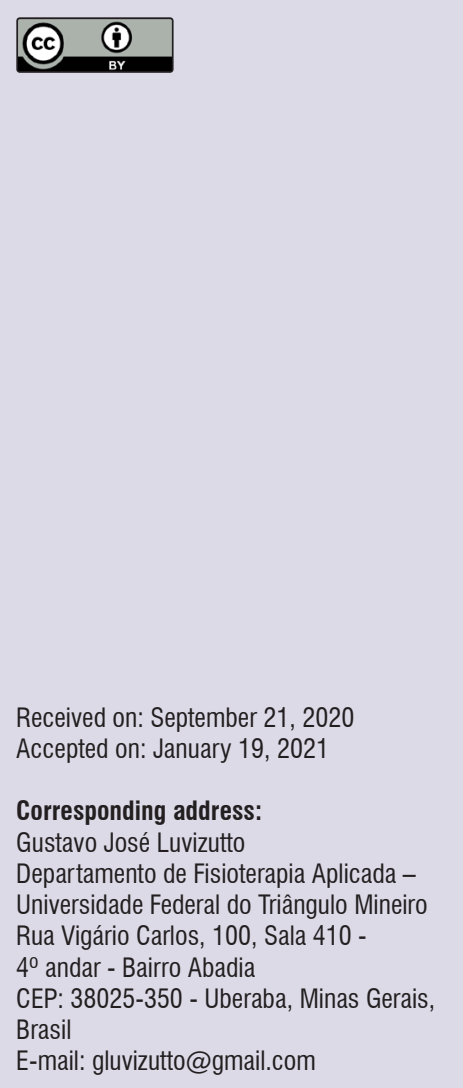

\section{ABSTRACT}

Purpose: this study aimed to verify the occurrence of abnormal vectoelectronystagmography findings in patients with temporomandibular disorders (TMDs).

Methods: in this cross-sectional study, patients diagnosed with TMDs underwent an otorhinolaryngological examination, audiological evaluation, and balance and vestibular function examinations, using vectoelectronystagmography. The tests performed were 1) spontaneous nystagmus, 2) saccadic movements, 3) pendular tracking, 4) optokinetic nystagmus gain and velocity, 5) rotational chair testing, and 6) post-caloric vertigo and the direction and velocity of the slow component of nystagmus.

Results: thirty patients were selected (22 females and 8 males) with mean age of $30.8 \pm 14.9$ years. Sensorineural hearing loss was seen in four patients (13.3\%); the other patients $(86.7 \%)$ had results within the normal range at all frequencies. Five patients (16.7\%) showed abnormalities on the Romberg test and seven (23.3\%) on the Tandem test. Abnormalities on the caloric test were seen in $40.0 \%$ of patients. More prevalence of headache $(p<0.0001)$ and tinnitus $(p<0.0001)$ was observed in patients with unilateral hyperreflexia, and dizziness, depression, anxiety, gait imbalance and falls in patients with bilateral hyperreflexia

Conclusion: patients with TMDs may present vectoelectronystagmography abnormalities characterized by unilateral or bilateral hyperreflexia and unilateral hyporeflexia of post-caloric nystagmus.

Keywords: Temporomandibular Disorders; Temporomandibular Joint; Labyrinth; Vestibular Function Tests; Nystagmus 


\section{INTRODUCTION}

Temporomandibular disorders (TMDs) are the second most common musculoskeletal conditions affecting the temporomandibular joint (TMJ), the jaw muscles, and/or related structures, and their etiology can be arthropathic, myofascial, or mixed ${ }^{1}$. A large proportion of the adult population may show some sign of TMD; however, the symptoms are manifested mainly in women'. The clinical picture is variable, with signs and symptoms of headache, periauricular pain, otalgia, fatigue in the jaw muscles, pain on palpation of the soft tissues, bruxism, pain in the cervical region and/or scapular girdle, and related psychological disorders ${ }^{3}$.

Several authors have suggested a relationship between TMD symptoms and alterations in the vestibular system, such as the presence of hypoacusis, aural fullness, tinnitus, and dizziness, resulting from the altered mechanics of the mandibular condyle of the temporal bone, which would compress the auditory tube, or from the relationship with tinnitus due to excessive contraction of the jaw muscles ${ }^{4}$. Several studies were conducted to understand the relationship between otoneurological symptoms and TMDs, and these studies showed that the head position influences the mandibular resting position, thereby increasing muscle tension ${ }^{5}$. In addition, most patients with tinnitus, headache, and dizziness presented abnormalities during mandibular movement ${ }^{6,7}$, dislocated articular disk $^{8}$, and abscesses or diseases of the dental pulp due to bruxism 9 .

The relationship between TMDs and vestibular disorders can also be attributed to the effects of drugs used in orthodontic or conventional therapies. The use of plaque repositioning and anti-inflammatory drugs in patients with TMD improved the symptoms of tinnitus and dizziness after 3 months of treatment ${ }^{10}$. In addition, researchers observed a relationship between the cervical spine and TMDs. Cervical spasms stimulate receptors causing changes in the cervical postural reflex, thereby increasing the tone of the jaw muscles. The increased excitability of the jaw muscles innervated by the trigeminal nerve can trigger mandibular parafunction and clenching of the teeth. The muscles of the soft palate may experience some interference, resulting in malfunction of the auditory tube and generating tinnitus. Moreover, the trigeminal hyperactivity in TMDs can disturb central vision; its constant stimulation changes the position of the head and interferes with the harmonious functioning of the superior colliculus involved in the regulation of visual fixation and vestibulo-ocular reflexes (VORs) ${ }^{11}$.

Currently, one of the most used methods for assessing VORs is digital vectoelectronystagmography. This examination can identify the exact direction of vestibulo-ocular phenomena, providing greater precision in the automatic measurement of the speed of the slow component of nystagmus, establishing more suitable proposals for therapy, and aiding the patient during rehabilitation treatment. The aim of this study was to verify the occurrence of abnormal findings on vectoelectronystagmography in patients with TMDs. The main hypothesis of this study was that patients with TMDs assessed, using vectoelectronystagmography, may present abnormalities in VORs.

\section{METHODS}

\section{Study Design, Setting, and Participants}

This cohort study included patients diagnosed with TMDs. The individuals were followed up at the Center of the Federal University of São Paulo, SP, Brazil. This study was approved by the Institutional Review Board (275/01). All participants provided their written consent.

\section{Inclusion Criteria}

Patients diagnosed with TMDs, according to the Research Diagnostic Criteria for Temporomandibular Disorders, were included with age over 18 years ${ }^{12}$.

\section{Exclusion Criteria}

Patients with ear, nose, and throat disorders were excluded by an otorhinolaryngology evaluation. Those with previous central or peripheral vestibular dysfunction, metabolic changes (hypothyroidism or diabetes mellitus), and associated neurological diseases were also excluded by consulting the patient's medical record and medical history.

\section{Proceedings}

The study was approved by the ethics and research committee of origin institution, and 33 patients with TMDs underwent anamnesis, otorhinolaryngological examination, audiological evaluation, and examinations of balance and vestibular function. All individuals (or their caregivers) involved in the study signed the Informed Consent Form. All individuals involved in the study signed the Informed Consent Form. 


\section{Variables}

The patients underwent comprehensive anamnesis about the signs and symptoms directly or indirectly related to otoneurological disorders ${ }^{13}$. The otorhinolaryngological examination was performed to exclude possible changes in the ears, nose, and throat that could interfere with the other tests.

The audiological evaluation was used to verify the integrity of the functional systems in the external, middle, and internal ears. Tonal and vocal audiometry was performed using the MA 41 audiometer $\left(\mathrm{MAICO}^{\circledR}\right)$, which was used to define the degree of hearing loss $^{14}$. The findings of tympanometric curves and stapedial reflexes determined using the AZ7 impedance device $\left(\right.$ MedWOW $^{\circledR}$ ) were also analyzed and classified ${ }^{15}$.

To perform the vestibular function tests, the patients were instructed to not use any medications for dizziness, stress or anxiety in the previous 72 hours, and to avoid tea, coffee, chocolate, soda, tobacco, and alcoholic beverages in the previous 48 hours, as these could interfere with eye movements and alter the examination results. On the day of the examination, the patients had a light meal, after maintaining a 3-hour fast. For investigating nystagmus and/or positional vertigo, the Dix-Hallpike and supine roll tests were performed ${ }^{16}$.

Vestibular function was assessed using digital vectoelectronystagmography. A VECWIN digital vectoelectronystagmograph, light bar, and NGR-05 air caloric stimulator (Neurograff Eletromedicina Ind. e Com. Ltda-EPT) were used for testing. Five supranuclear oculomotor systems were analyzed: smooth pursuit, saccadic, vestibular, optokinetic, and rapid refixation movements. Positioning nystagmus was investigated by seating the patient, placing the patient's head at a 45-degree angle to one side, rapidly reclining the patient to the lateral decubitus position on the opposite side, maintaining the position, rapidly moving the patient back to the seated position, and then repeating the maneuver with the patient's head angled 45 degrees to the other side. Each position was maintained for 30 seconds or until dizziness and/or nystagmus yielded or disappeared. Before the test, the patients were instructed to not oppose any movement and to not close their eyes.

Thereafter, the face skin was cleaned and three active electrodes and one ground electrode were placed before performing digital vectoelectronystagmography. Three deriving active electrodes were placed on the right and left external periorbital angles and on the frontal midline to record eye movements along three recording channels. Calibration was performed to ensure similar conditions for the various steps of the test and to automatically record latency, precision, velocity, and gain for eye movements other than the slow component of nystagmus ${ }^{17}$.

The following tests were performed using vectoelectronystagmography:

1. Spontaneous nystagmus was investigated in the central gaze with the eyes open and closed. Semi-spontaneous nystagmus was investigated in right, left, upward, and downward gaze deviation not more than 30 degrees from the midline. The slow component velocity was measured during the test.

2. Saccadic movements were assessed by visually following a regularly or randomly moving target. Saccade latency, velocity, and precision were also assessed.

3. Pendular tracking was assessed by having the patient visually follow the sinusoidal movement of a light target at $0.2-$ and $0.4-\mathrm{Hz}$ frequencies. Eye movement type and gain were assessed in this test.

4. Optokinetic nystagmus gain and velocity were measured by having the patient visually follow a moving light target in a fixed direction to one and then to the other side at $10 \%$ s.

5. Rotational chair testing was performed with the patients seated with their eyes closed. Then the head was tilted forward at a 30-degree angle to stimulate the lateral semicircular canals and tilted backwards at a 60-degree angle to stimulate the posterior and superior semicircular canals. Thereafter, the head was tilted 45 degrees to the right, 60 degrees backwards, and 45 degrees to the left. The chair was rotated 90 degrees from the center and released, thereby resulting in a decrease in amplitude periodic pendular movement. The resulting peri-rotational nystagmus was assessed by measuring the velocity of its slow component. Pre-rotational nystagmus and its influence on the test results were also investigated.

6. Caloric testing was performed by stimulating each ear separately with air. Post-caloric vertigo and the direction and velocity of the slow component of nystagmus were assessed with the eyes open and closed. Pre-caloric nystagmus and its influence on the test results were also investigated. 


\section{Data Analysis}

Data analysis was performed using the first vectoelectronystagmography channel. The results of each patient were automatically compared with the normal limits in each test, thus allowing an immediate assessment of possible vestibular functional abnormalities. The data of the included patients were analyzed separately as well as together, in order to identify possible signs of dysfunction of the vestibular system. The Fisher's exact test was performed to compare the prevalence of symptoms between patients with abnormal findings in the vectoelectronystagmography. The associations were considered statistically significant if $p$ was $<0.05$. Data analysis was performed using GraphPad Prism Version 8.3.1 software.

\section{RESULTS}

Thirty-three patients were evaluated, and 30 of them were selected for the vestibular test. One patient with hypothyroidism and two with diabetes mellitus were excluded. The selected individuals were aged between 15 and 60 years; 22 were females and 8 were males. The average age of the individuals was $30.8 \pm 14.9$ years.

Audiological evaluation revealed high-frequency bilateral sensorineural hearing loss in four patients (13.3\%). The other patients (86.7\%) presented values within the normal range at all frequencies. The symptoms found in the anamnesis are listed in Table 1.

Table 1. Symptoms of the included patients $(n=30)$

\begin{tabular}{lcc}
\hline Variable & N & $\%$ \\
\hline Sleep disorders & 28 & 93.3 \\
Headache & 24 & 80.0 \\
Neck crackles & 20 & 66.7 \\
Neck pain & 17 & 56.7 \\
Fatigue & 14 & 46.7 \\
Tinnitus & 12 & 40.0 \\
Dizziness & 10 & 33.3 \\
Depression & 10 & 33.3 \\
Anxiety & 9 & 30.0 \\
Otalgia & 8 & 26.6 \\
Pallor & 7 & 23.3 \\
Vomiting & 7 & 23.3 \\
Paresthesia & 7 & 23.3 \\
Nausea & 6 & 20.0 \\
Radiating pain & 5 & 16.6 \\
Hypoacusis & 5 & 16.6 \\
Gait imbalance & 4 & 13.3 \\
Vision dimming & 3 & 10.0 \\
Falls & 2 & 6.7 \\
\hline
\end{tabular}

In the Romberg static balance test, abnormalities were observed in five individuals (16.7\%), and in the Tandem test, abnormalities were observed in seven (23.3\%). No changes were found in the Dix-Hallpike and supine roll tests.
In the caloric test, 12 patients (40.0\%) presented with abnormalities. Table 2 shows the classification of abnormal vestibular responses to air labyrinthine stimulation. 
Table 2. Prevalence of abnormal findings in the angular velocity of the slow component of post-caloric nystagmus in 30 patients with temporomandibular disorders, given in degrees per second

\begin{tabular}{lcc}
\hline Abnormal finding & N & $\%$ \\
\hline Unilateral hyperreflexia & 7 & 23.3 \\
Bilateral hyperreflexia & 3 & 10.0 \\
Unilateral hyporeflexia & 2 & 6.6 \\
\hline Total & 12 & 40.0 \\
\hline
\end{tabular}

The symptoms of 12 patients with abnormal findings in the angular velocity of the slow component of post-caloric nystagmus are showed in Table 3. More prevalence of headache $(p<0.0001)$ and tinnitus $(p<0.0001)$ was observed in patients with unilateral hyperreflexia. The prevalence of dizziness, depression, anxiety, gait imbalance and falls were observed in patients with bilateral hyperreflexia

Table 3. Symptoms of 12 patients with abnormal findings in the angular velocity of the slow component of post-caloric nystagmus

\begin{tabular}{lcccc}
\hline Variable & Unilateral hyperreflexia & Bilateral hyperreflexia & Unilateral hyporeflexia & p values \\
\hline Sleep disorders & $4(57.14 \%)$ & $2(66.67 \%)$ & $1(50.0 \%)$ & 0.07 \\
Headache & $7(100.0 \%)$ & $1(33.33 \%)$ & $1(50.0 \%)$ & $<0.0001$ \\
Tinnitus & $7(100.0 \%)$ & $1(33.33 \%)$ & $0(0 \%)$ & $<0.0001$ \\
Dizziness & $6(85.71 \%)$ & $3(100.0 \%)$ & $2(100.0 \%)$ & $<0.0001$ \\
Depression & $4(57.14 \%)$ & $3(100.0 \%)$ & $2(100.0 \%)$ & $<0.0001$ \\
Anxiety & $5(71.43 \%)$ & $2(66.67 \%)$ & $1(50.0 \%)$ & 0.005 \\
Gait imbalance & $2(28.57 \%)$ & $3(100.0 \%)$ & $2(100.0 \%)$ & $<0.0001$ \\
Falls & $0(0 \%)$ & $3(100.0 \%)$ & $1(50.0 \%)$ & $<0.0001$ \\
\hline
\end{tabular}

\section{DISCUSSION}

This study demonstrates the effects of TMDs on the vestibular system. According to the results, the five most prevalent symptoms were that $93 \%$ had sleep disturbance, $80 \%$ of patients had headache, $66 \%$ had neck cracking, $56 \%$ had neck pain, and $46 \%$ had fatigue. The high prevalence of headache in patients with TMDs has been observed in several studies in the literature, and the following factors are frequently associated with it: females, age over 25 years, sleep disorders, and arthritis or cervical arthrosis with joint and muscle involvement ${ }^{18,19}$. A study also reported that the loss of the centric relationship of the cervical spine leads to the absence of the centric relationship of the TMJ, resulting in craniomandibular disorders, excessive muscle activity, and the occurrence of headache and local pain ${ }^{20}$.

The abnormal audiological findings of the present study were compatible with the diagnostic hypothesis of presbycusis. A previous investigation showed sensorineural hearing loss in six recruited patients (16.7\%) with
TMD ${ }^{21}$. Previous studies have also reported a functional correlation between the masticatory and auditory systems. Hypertonia in the masticatory muscles generates reflex contraction of the tensor tympani and tensor palatini muscles. These muscles present anatomical proximity to the muscles involved in TMDs, in addition to being innervated by the same mandibular branch of the trigeminal nerve. Thus, hypertonia in this musculature impairs the opening of the auditory tube, which causes pressure in the ears (aural fullness), imbalance, and hearing loss. Moreover, spasms of the tensor tympani muscle cause the same symptoms, in addition to otalgia, tinnitus, and headache ${ }^{6}$.

When present, vertigo was associated with other forms of dizziness in 10 patients (33.3\%) and only with non-rotating dizziness in 4 patients. Digital vectoelectronystagmography demonstrated that 12 patients $(40.0 \%)$ presented changes in vestibular function. These data demonstrate that several symptoms may be related to TMDs as well as the impairment of the auditory and vestibular systems. The presence of these 
patients suggests a relationship involving the vestibular and stomatognathic systems ${ }^{23}$. The stomatognathic system also plays an important role in postural control. Patients with TMDs show greater changes in the body's center of gravity ${ }^{22}$. These anatomical connections suggest that portions of the trigeminal system strongly influence the coordination of posture and sight. Sensory information from proprioceptive receptors in the stomatognathic system is likely processed in the Tandem test with information from the vestibular and oculomotor systems. Changes in trigeminal stimulations can cause an imbalance in the vestibular and oculomotor systems ${ }^{22}$.

The caloric test was the only one whose results showed variations in the study population. Ten patients presented hyperreflexia compatible with the hypothesis of peripheral vestibular syndrome - seven unilateral and three bilateral. Two patients presented deficient peripheral vestibular syndrome due to a decreased response. A previous study reported 20 patients with TMDs (74.0\%) who showed changes on vectoelectronystagmography, including 6 (22.2\%) with directional preponderance of nystagmus, 5 (18.5\%) with unilateral labyrinthine hyporeflexia, 4 (14.8\%) with bilateral labyrinthine hyporeflexia, $3(11.1 \%)$ with unilateral labyrinthine hyperreflexia, 2 (7.4\%) with asymmetric labyrinthine preponderance, and 7 (26.0\%) with normoreflexia ${ }^{23}$.

The patients with unilateral hyperreflexia it was observed more prevalence of headache and tinnitus, and the more prevalence of dizziness, depression, anxiety, gait imbalance and falls were observed in patients with bilateral hyperreflexia. Otological alterations unrelated to the peripheral or central labyrinthic lesion might generate hyperreflexia ${ }^{24}$. In peripheral vestibulopathies, hyperreflexia can be found on the side contralateral to the labyrinth with a deficient response. The vestibular change in patients with TMDs can be explained by the inflammatory process, which increases the activity of the ipsilateral vestibular nucleus; this imbalance is responsible for the production of post-caloric nystagmus in the examination ${ }^{25}$.

The main limitations of the present study are the small sample size and the absence of a control group. However, the aim of this study was to highlight the importance of assessing the vestibular system in individuals with TMDs who report otoneurological symptoms. These findings justify the systematic inclusion of otoneurological investigation in patients with TMDs to verify diagnostic, prognostic, and therapeutic implications.
Vectoelectronystagmography has proven effective in identifying abnormalities of post-caloric nystagmus in a relevant proportion of patients with TMDs. This is an important finding from an otoneurological perspective, since it clarifies the relationship between the vestibular and temporomandibular systems.

\section{CONCLUSION}

Based on our results, patients with TMDs may present abnormalities on vectoelectronystagmography, characterized by unilateral or bilateral hyperreflexia and unilateral hyporeflexia of post-caloric nystagmus, in a decreasing order of prevalence.

\section{REFERENCES}

1. Fernández-de-las-Penas C, Svensson P. Myofascial temporomandibular disorder. Curr Rheumatol Rev. 2016;12(1):40-54.

2. Maixner $W$, Diatchenko $L$, Dubner R, Fillingim RB, Greenspan JD, Knott C et al. Orofacial pain prospective evaluation and risk assessment study -- the OPPERA study. J Pain. 2011;12(11 suppl):T4-T11.e1-2.

3. Durham J, Newton-John TR, Zakrzewska JM. Temporomandibular disorders. BMJ. 2015;350:h1154.

4. Edvall NK, Gunan E, Genitsaridi E, Lazar A, Mehraei $G$, Billing $M$ et al. Impact of temporomandibular joint complaints on tinnitus-related distress. Front Neurosci. 2019;13:879.

5. Scharnweber B, Adjami F, Schuster G, Kopp $S$, Natrup J, Erbe $C$ et al. Influence of dental occlusion on postural control and plantar pressure distribution. Cranio. 2017;35(6):358-66.

6. Villaça Avoglio JL. Dental occlusion as one cause of tinnitus. Med Hypotheses. 2019;130:109280.

7. Bousema EJ, Koops EA, van Dijk P, Dijkstra PU. Association between subjective tinnitus and cervical spine or temporomandibular disorders: a systematic review. Trends Hear. 2018;22:2331216518800640.

8. Skog C, Fjellner J, Ekberg E, Häggman-Henrikson B. Tinnitus as a comorbidity to temporomandibular disorders-A systematic review. J Oral Rehabil. 2019;46(1):87-99.

9. Vielsmeier V, Strutz J, Kleinjung $T$, Schecklmann $M$, Kreuzer PM, Landgrebe $M$ et al. Temporomandibular joint disorder complaints in tinnitus: further hints for a putative tinnitus subtype. PLoS One. 2012;7(6):e38887. 
10. Wright EF, Bifano SL. The relationship between tinnitus and temporomandibular disorder (TMD) Therapy. Int Tinnitus J. 1997;3(1):55-61.

11. Boniver R. Temporomandibular joint dysfunction in whiplash injuries: association with tinnitus and vertigo. Int Tinnitus J. 2002;8(2):129-31.

12. Dworkin SF, LeResche L. Research diagnostic criteria for temporomandibular disorders: review, criteria, examinations and specifications, critique. J Craniomandib Disord. 1992;6(4):301-55.

13. Guzmán PV, Caovilla HH. Sintomas otoneurológicos: investigação por meio de um questionário. Acta AWHO. 2001;20(3):130-40.

14. Davies H. Audiometry: pure-tone and simple speech test. In: Davis H, Silverman R (eds). Hearing and deafness. New York: Holt, Rinehart and Winston; 1978. p. 183-221.

15. Jerger J. Clinical experience with impedance audiometry. Arch Otolaryngol. 1970;92(4):311-24.

16. Cohen HS. A review on screening tests for vestibular disorders. J Neurophysiol. 2019;122(1):81-92.

17. Pansini M, Padovan I. Three derivations in electronystagmography. Acta Otolaryngol. 1969;67(2):303-9.

18. Martin VT, Neilson D. Joint hypermobility and headache: the glue that binds the two together-part 2. Headache. 2014;54(8):1403-11.

19. Di Paolo C, D'Urso A, Papi P, Di Sabato F, Rosella $D$, Pompa $G$ et al. Temporomandibular disorders and headache: a retrospective analysis of 1198 patients. Pain Res Manag. 2017;2017:3203027.

20. La Touche R, París-Alemany A, von Piekartz $\mathrm{H}$, Mannheimer JS, Fernández-Carnero J, Rocabado M. The influence of cranio-cervical posture on maximal mouth opening and pressure pain threshold in patients with myofascial temporomandibular pain disorders. Clin $\mathrm{J}$ Pain. 2011;27(1):48-55.

21. Manni A, Brunori $P$, Giuliani $M$, Modoni $M$, Bizzi G. Oto-vestibular symptoms in patients with temporomandibular joint dysfunction. Electromyographic study. Minerva Stomatol. 1996;45(1-2):1-7.

22. Cuccia A, Caradonna C. The relationship between the stomatognathic system and body posture. Clinics. 2009;64(1):61-6.

23. Zeigelboim BS, Jurkiewicz AL, Martins-Bassetto J, Klagenberg KF. Avaliação vestibular em mulheres com disfunção temporomandibular. Rev. CEFAC. 2007;9(2):255-62.
24. Gonçalves DU, Felipe L, Lima TM. Interpretation and use of caloric testing. Braz J Otorhinolaryngol. 2008;74(3):440-6.

25. Chung SW, Kim MJ, Ahn JC, Suh MW, Rhee CK, Jung JY. Inflammation of temporomandibular joint increases neural activity in rat vestibular nucleus. Neurosci Lett. 2012;528(2):126-30. 